\title{
Clinical significance of umbilical region involvement in pemphigus vulgaris in a series of 81 ethnic Poles: a comparative analysis of the distribution of lesions in two infrequent locations
}

\author{
Magdalena Jałowska ${ }^{1}$, Justyna Gornowicz-Porowska ${ }^{1,2}$, Agnieszka Seraszek-Jaros ${ }^{3}$, Monika Bowszyc-Dmochowska ${ }^{4}$, \\ Elżbieta Kaczmarek ${ }^{3}$, Marian Dmochowski ${ }^{1}$
}

\begin{abstract}
${ }^{1}$ Autoimmune Blistering Dermatoses Section, Department of Dermatology, Poznan University of Medical Sciences, Poznan, Poland ${ }^{2}$ Department and Division of Practical Cosmetology and Skin Diseases Prophylaxis, Poznan University of Medical Sciences, Poznan, Poland

${ }^{3}$ Department of Bioinformatics and Computational Biology, Poznan University of Medical Sciences, Poznan, Poland

${ }^{4}$ Cutaneous Histopathology and Immunopathology Section, Department of Dermatology, Poznan University of Medical Sciences, Poznan, Poland
\end{abstract}

Adv Dermatol Allergol 2022; XXXIX (2): 281-285

DOI: https://doi.org/10.5114/ada.2021.102857

\begin{abstract}
Introduction: Autoimmune bullous diseases are potentially life-threatening dermatoses which present with cutaneous and/or mucosal blisters, diagnosed on the basis of clinical manifestations, direct immunofluorescence of perilesional tissue, and serum testing for circulating autoantibodies. Sometimes, lesions in the navel can lead to the diagnosis of a bullous disease.

Aim: To assess the frequency of occurrence of pemphigus lesions located in the navel area and nail apparatus in pemphigus vulgaris (PV) in ethnic Poles.

Material and methods: Eighty one patients (31 males and 50 females, mean age 59 years) with dermatoses of the PV group diagnosed in 2002-2020 were retrospectively analysed using their photographic files. Statistical analysis was performed using the difference test between two proportions to check the difference between the percentage of PV patients with navel area involvement and nail apparatus involvement.

Results: There was no statistically significant difference between PV patients with nail apparatus involvement $(12.3 \%)$ and navel area involvement $(14.8 \%)(p=0.4632)$. Only females had lesions in the navel area in our series of PV patients.

Conclusions: It is speculated that the causal relationship may exist between the female reproductive system and the pattern of expression of PV lesions around the umbilicus. The awareness that PV can infrequently affect the umbilical region and the nail apparatus should help in some cases to establish the diagnosis of PV. The periumbilical involvement can facilitate the performance of DIF in individuals with lesions in less accessible areas.
\end{abstract}

Key words: umbilicus, umbilical lesions, pemphigus vulgaris.

\section{Introduction}

Pemphigus diseases are relatively rare, but form a potentially life-threatening group of autoimmune blistering dermatoses (ABD) in which immunoglobulin $G$ (IgG) antibodies target desmosomal proteins to produce intraepithelial, mucocutaneous blistering. Diagnosis relies on a combination of clinical features as well as the direct immunofluorescence of perilesional tissue and se- rum testing with molecular-biochemical techniques for autoantibodies to desmogleins [1]. The pemphigus group presents chronic evolution, with significant mortality and an important impairment in quality of life. Characteristic skin lesions of pemphigus mainly are located at the transition between the epithelium/extra-residual epidermis and the follicular epithelium - the scalp; trunk anterior and posterior seborrheic areas, as well as in places of transition between the epidermis and stratified squa-

Address for correspondence: Prof. Marian Dmochowski MD, PhD, Autoimmune Blistering Dermatoses Section, Department of Dermatology, Poznan University of Medical Sciences, 49 Przybyszewskiego St, 60-355 Poznan, Poland, phone: +48 618691319 , e-mail: mdmochowski@ump.edu.pl

Received: 28.11.2020, accepted: 31.12.2020. 
mous epithelium of mucous membranes - around natural body orifices [2]. It is also suggested that the skin appendages (hair follicles, eccrine glands, nail apparatus) are affected by processes occurring in the course of pemphigus diseases [2]. It is believed that the nail involvement in pemphigus vulgaris (PV) is uncommon; however, literature data indicate the presence of lesions there in up to $22-30 \%$ of PV patients [3]. The temporal relationship with the occurrence of nail lesions in PV varies: it may be a part of the primary manifestation, accompany a relapse, or be a harbinger of its exacerbation. Moreover, it is suggested that the appearance of haemorrhagic nail eruptions in the course of PV, related to the severity of the disease, may be useful in the prognostic assessment of the patient's condition [4]. It is also interesting that nail apparatus involvement may be the only indicator of the disease process at the beginning of PV. The study of photographic archives from 2002-2017 in ethnic Poles conducted in a setting of a Central European university dermatology department and assessing exclusively nail apparatus changes showed that such lesions were present in just $13.4 \%$ of PV patients, namely they were less frequent than in other ethnicities [4].

The umbilicus (navel, belly button) is a scar on the abdomen caused by the removal of the umbilical cord from the newborn baby. It is the central relatively weak depression of the anterior abdominal wall opposite the disk between $L 3-L 4$ vertebrae or L4 vertebra [5]. It is an important aesthetic component of the body. Its abnormal appearance or absence may cause distress, may even be a source of grief and depression. Across cultures umbilicus has been not only a sign of youth and beauty, but also the source of sexual arousal epitomized by belly dancing. Extraordinarily, the comprehensive survey of belly-button lint conducted by Kruszelnicki of the University of Sydney, won the Ig Nobel Prize for Interdisciplinary Research [6]. Navel stones can even form there. The first known use of the word navel was before the $12^{\text {th }}$ century [7]. The word is often used in telling collocations, such as the navel of the world, navel-gazing. As such, an aesthetically pleasing umbilicus is regarded as necessary for an attractive abdomen. Navel fetishism or belly button fetishism is a partialism in which an individual is attracted to the human navel. In the 1960s or 1970s navel piercings have become quite common in Western societies in order to draw attention to the navel.

Umbilical lesions are rare but always require detailed diagnostics to rule out hidden malignancy [8] and should be diagnosed on careful clinical examination and histopathological evaluation. In some cases, the direct immunofluorescence (DIF) examination is of key importance. Umbilical lesions as a clinical manifestation of pemphigus have been reported very rarely so far, but already in the textbook of Grzybowski titled "Skin Diseases for Doctors and Students" from 1948, the navel was indicated as an important site of involvement in pemphigus vegetans (PVEG) [9].
The navel area as one of the predilection sites in PVEG is also described in the Japanese dermatological textbook for medical students [10]. Recognition of this presentation is relevant. The differential diagnosis should be made with pyogenic granuloma, erythema multiforme, psoriasis, impetigo, intestinal fistulae and metastatic nodules and others. Possible explanation for the presence of pemphigus lesions in umbilicus include expression of altered antigens, linked with embryonic structures such as the umbilical cord that would stimulate IgG autoimmunity against desmogleins [11].

\section{Aim}

The aim of this study was to analyse the involvement of the umbilical region in PV in ethnic Poles and compare frequency of occurrence of pemphigus skin lesions located in the navel area and nail apparatus.

\section{Material and methods}

The study was conducted in a setting of a Central European university dermatology department. Eighty one Slavic patients ( 50 females and 31 males, female to male ratio $=1.61)$ with diseases of the PV group having IgG antibodies to DSG3 or both DSG3 and DSG1 were retrospectively analysed for the presence of umbilical and nail apparatus pemphigus lesions using photographic archives from 2002-2020. The study group consisted of 57 patients with mucocutaneous PV (MCPV), 17 with mucosal dominant PV (MPV), 3 patients with cutaneous PV (CPV), and 4 with PVEG. The age of the patients with pemphigus ranged from 31 to 90 years. The mean age was 59 years. The diagnoses were based on the combination of clinical data, and DIF of perilesional tissue also for evaluation of IgG4 deposits (Figure 1) having dew drops on spider web appearance [12] and IgG1 deposits, as well as mono-analyte ELISA with DSG1/3 (Euroimmun, Germany or MBL, Japan) or multi-analyte ELISA with envoplakin, type VII collagen, DSG1, DSG3, BP180, BP230 (Euroimmun, Germany) corroborated by the histopathological $\mathrm{H}+\mathrm{E}$ examination.

\section{Statistical analysis}

Statistical analysis was performed using Statistica, version 13 (StatSoft, Inc., Tulsa, OK, USA). Statistical significance was $\alpha=0.05$. A result was deemed statistically significant if $p<\alpha$. The difference test between two proportions was used to check the difference between the percentage of PV patients with navel area involvement and nail apparatus involvement.

\section{Results}

The nail apparatus involvement was found in 10 of 81 $\mathrm{PV}$ patients (12.3\%). Skin lesions in the navel area were 

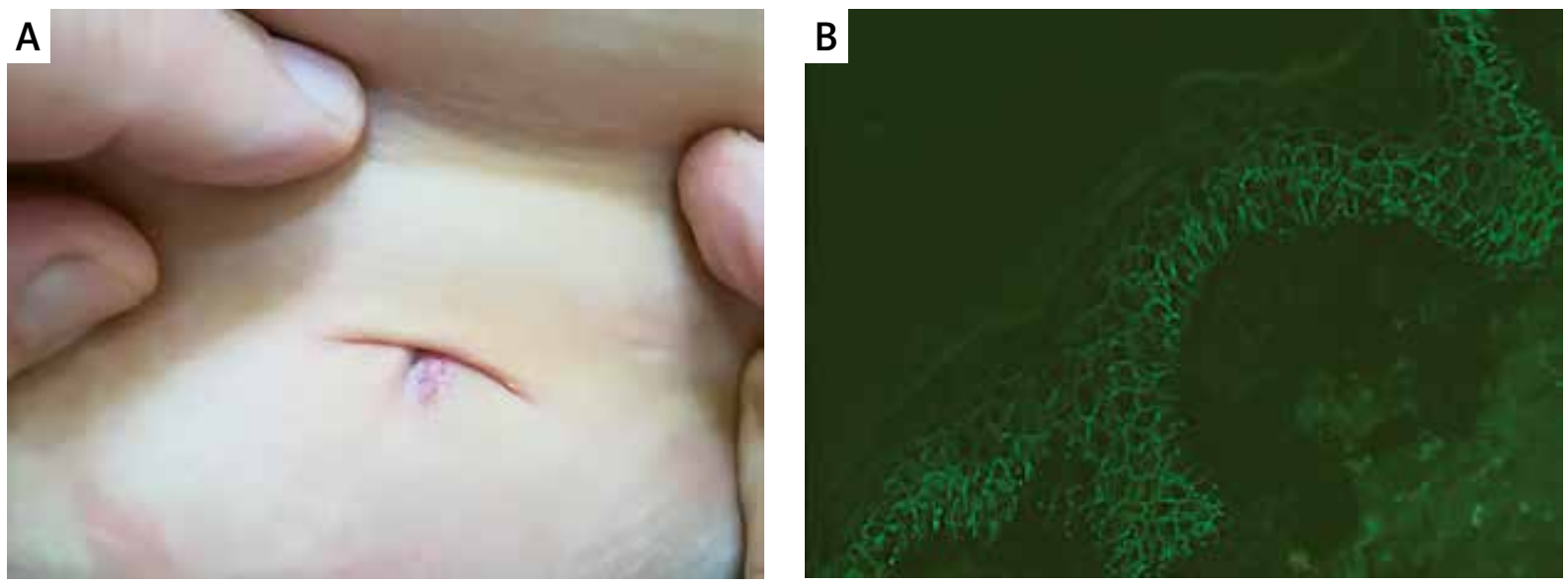

Figure 1. A 63-year-old female with mucocutaneous pemphigus vulgaris affecting also the umbilical region (A). Direct immunofluorescence of perilesional skin around the navel showed pemphigus intercellular IgG4 (+++) deposits having dew drops on spider web appearance (B) and C3 (+) deposits. Multi-analyte ELISA revealed elevated levels of IgG serum anti-DSG1 (1.8) and anti-DSG3 (5.46) antibodies (cut-off values below 1.0). The lesions on the trunk were surgically removed without any clinical suspicion of pemphigus vulgaris and sent for histopathological H+E examination. The pathologist reported that the picture suggested pemphigus and only then the patient was referred to a dermatologist dealing with autoimmune blistering dermatoses

present in 12 of $81 \mathrm{PV}$ patients (14.8\%) and their assessment, limited by our study design involving an analysis of just photographic files, was described here. Only females (10 with MCPV, 1 with MPV and 1 with PVEG) had lesions in the navel area (Figure 1). There was no significant difference between PV patients with nail apparatus involvement and navel area involvement ( $p=0.4632$ ). One young female patient with MPV presented with just a single cutaneous lesion in the umbilical region, and the biopsy for DIF was taken from that area. Initially, the patient had erosions on the oral mucosa, and neither the patient nor the family practitioner were aware that it is the same disease. One middle-aged female patient with MCPV exhibited lesions both around the navel and in the nail apparatus.

\section{Discussion}

Skin lesions around the umbilical region always require a detailed differential diagnosis. Differentiation should take into account not only infectious diseases, neoplastic diseases, inflammatory diseases including psoriasis, allergic diseases and others, but also autoimmune bullous diseases. In the case of lesions in the navel area, it is extremely rare to think of blistering diseases, pregnant/lactating females are the exception. In pemphigoid gestationis (PG), skin lesions typically develop on the abdomen. Blisters characteristically occur in the umbilical region and later spread to the rest of the body. The first immune response in PG occurs within the placenta because placental trophoblasts and amniochorionic stromal cells show an abnormal expression of major histocompatibility complex (MHC) class II antigen [13]. It is striking that only females with PV had lesions in the navel area in our study. This observation may be just fortuitous as our study was designed to analyse retrospectively photographic archives merely in a single tertiary referral centre, but we are tempted to speculate that the causal relationship may exist between the female reproductive system and the pattern of expression of PV lesions around the umbilicus. The microchimerism may underlie this connection. Those assumptions require further analysis ideally on different series of PV patients using a multicentre approach.

Some publications state that the umbilical area is a predilection site for patients with a "classical" variety of bullous pemphigoid (BP) [14]. Schmidt et al. described $\mathrm{BP}$ with the skin lesions limited only to the umbilical region [15]. Sometimes lesions in this cutaneous location may appear initially in ABD and therefore the umbilical area is diagnostically valuable. This peculiar presentation in pemphigus, not yet completely elucidated, has been rarely reported in the literature. Umbilical region involvement in patients with pemphigus was assessed in a limited study totalling just 10 patients [16]. A possible explanation may be the presence of either novel epitopes or an association with embryonic or scar tissue located in the umbilical-cord region [16]. Erythema, erosions, crusts and vegetating skin lesions were the main clinical features of pemphigus of the umbilical region. Such skin lesions around the navel most often suggest a nickelrelated allergic contact dermatitis (ACD). The development of an itchy eczematous eruption near the umbilicus is almost pathognomonic for contact dermatitis to nickel. There are two reports in the literature which suggest that nickel can induce pemphigus-type lesions $[17,18]$. Th17 lymphocytes which are involved in the pathogenesis of both ACD and pemphigus may be responsible for this 
process. Indications for involvement of Th17 in human skin allergy were reported for the first time by detection of IL-17 mRNA in skin lesions from nickel allergic patients and in skin-derived nickel specific T cells [19]. In several human studies, T-cell-clones could be isolated from eczema biopsies, and high IL-17 levels were observed after challenge with an allergen [19]. A crucial role of Th17 in ACD is recruitment of neutrophils, activation of fibrocytes and macrophages. The indications for the participation of Th17 in the development of ACD are supported by data from IL-17 deficient mice with reduced contact hypersensitivity reactions that could be restored after transplantation of wild type CD4+T cells [20]. Also PV patients had higher serum levels of inflammatory Th1/Th17 cytokines (IFN- $\gamma, \mathrm{IL}-17$, and IL-23) than in controls [21]. Psoriasis is also a disease with an autoimmune background, in which Th17 cells and IL-17 are important for pathogenesis. Of note, psoriatic lesions are very often located around the navel. Furthermore, it was reported that there are female DIF-proven dermatitis herpetiformis $(D H)$ sufferers in whom a coexistent ACD to nickel as detected by epicutaneous patch tests with $5 \%$ nickel sulfate hexahydrate in Vaseline can be found [22]. It was speculated there that IL-6, a pleiotropic cytokine, can link pathogenesis of ACD to nickel and $\mathrm{DH}$. According to our clinical-laboratory-research experience, DH can affect the navel area undergoing a prolonged microtrauma by a waist belt. Thus, the navel involvement in a plethora of apparently diverse dermatoses may simply reflect the fact that they share pathological traits.

Another reason for the appearance of skin lesions around the navel could be a Koebner phenomenon (KP) defined as the appearance of new blisters or erosions days after the skin damage by the ACD. There have been few case reports of new pemphigus lesions developing at the sites of surgical procedures and burns. Daneshpazhooh et al. presented a patient who developed new pemphigus lesions in the umbilical region following umbilical-urachal surgery [23]. The pathogenesis of KP in PV is unknown and probably involves a multifactorial interaction [23]. Early development of blisters (during the first days) may be considered to be mediated by proinflammatory mediators, released in a nonspecific manner following injuries, through non-antigen-specific mechanisms by modulating C3, plasminogen activator, and plasminogen activator inhibitor expression [24]. These elevated cytokines may act as facilitators in the already pemphigus-prone skin [23]. Moreover, a disruption of the skin's intactness has been suggested to allow for the exposure of pemphigus antigens, sufficient to react with lower titres of circulating antibodies that do not cause the disease in the absence of skin damage [23]. In the literature, we find just a few descriptions of skin lesions around the navel in paraneoplastic pemphigus $[25,26]$ and a peculiar presentation of PVEG in the umbilicus [27, 28].
Uruguayan authors described a case of pemphigus in a 2-year-old child with maximum lesion areas in the umbilical region, in limbs and genitals [29]. Umbilical pemphigus can be a rare clinical presentation of fogo selvagem, an endemic form of pemphigus foliaceus; it is characterized by erosions or vegetating lesions on the umbilicus, resembling intestinal fistula or pyogenic granuloma [30].

Prospective analysis of an astonishingly high number of 1209 patients diagnosed and followed at the Pemphigus Research Unit, Tehran University for Medical Sciences, from 1984 to 2003 was published [31]. The initial localization of the lesions were oral in 750 patients (62\%), limited to the scalp 161 (13\%), umbilical in 38 (3\%), and generalized in 36 (3\%) cases. In our study in ethnic Poles, skin lesions in the navel area occurred in $14.8 \%$ of PV patients at the time of presentation. Our comparative study indicates that both nail and umbilical areas are rarely affected in PV in ethnic Poles; yet the presence of lesions in those locations may be a hint [4] suggesting the proper diagnosis at the clinical level. Importantly, the periumbilical biopsy for DIF (Figure 1), the crucial procedure for in-depth diagnosing of pemphigus, can be more easily taken compared to mucous membranes or scalp. Finally, despite being infrequent, PV lesions around umbilicus and in the nail apparatus can drastically reduce the sensual-sexual wellbeing of the patients. Physicians attending PV patients should be aware of those psychological disturbances that may be aggravated by patients' feeling that their bodies are not as attractive as those promoted by aggressive advertising campaigns in the media.

\section{Conclusions}

It is speculated that the causal relationship may exist between the female reproductive system and the pattern of expression of PV lesions around the umbilicus. The diseases of the PV group of dermatoses should be kept in mind in the differential diagnosis at the clinical level of skin lesions in the umbilical region. In patients showing periumbilical lesions thorough immunopathological examinations as a part of differentiating laboratory work-up may be required for the definitive PV diagnosis. The periumbilical involvement can facilitate the performance of DIF in individuals having also lesions in less accessible areas. Subsequent proper management of PV should be also aimed at dealing with any psychological anxieties that may arise as a result of the umbilical region involvement even if the patient does not explicitly express such worries.

\section{Acknowledgments}

A part of this study was presented at the Online Conference "Kontrowersje w dermatologii 2020/Controversies in Dermatology 2020" by Monika Bowszyc- 
Dmochowska and titled "Pęcherzyca bujająca: czy jest wyzwaniem dla klinicysty?/Pemphigus vegetans: a challenge for the clinician?", 17-19 December 2020, Wrocław, Poland. This study was also undertaken within the framework of activities of the European Reference NetworkSkin.

\section{Conflict of interest}

The authors declare no conflict of interest.

\section{References}

1. Saschenbrecker S, Karl I, Komorowski L. Serological diagnosis of autoimmune bullous skin diseases. Front Immunol 2019; 20: 1974.

2. Dmochowski M. Natural body orifices and mucous membranes in pemphigus vulgaris. Dermatol Klin 2007; 9: 124-31.

3. Carducci M, Calcaterra R, Franco G, et al. Nail involvement in pemphigus vulgaris. Acta Derm Venereol 2008; 88: 58-60.

4. Pietkiewicz P, Bowszyc-Dmochowska M, Gornowicz-Porowska J, et al. Involvement of nail apparatus in pemphigus vulgaris in ethnic Poles is infrequent. Front Med 2018; 5: 227.

5. Hegazy AA. Anatomy and embryology of umbilicus in newborns: a review and clinical correlations. Front Med 2016; 10.

6. Brown C. IgNobel (3): navel-gazing. CMAJ 2002; 167: 1350.

7. https://www.merriam-webster.com/dictionary/ (Available at: 28.11.2020).

8. Das A. Umbilical lesions: a cluster of known unknowns and unknown unknowns. Cureus 2019; 11: e5309.

9. Grzybowski M. Skin diseases. A handbook for doctors and students. Lekarski Instytut Naukowo-Wydawniczy, Warsaw 1948; 182.

10. Ueno K. Minor Textbook Dermatology. Kinpodo, Kyoto 1991, 201-11.

11. Aoki W, Maruta C, Santi C. Umbilical involvement in pemphigus vulgaris. J Am Acad Dermatol 2010; 62: AB79.

12. Dmochowski M, Gornowicz-Porowska J, Bowszyc-Dmochowska M. Dew drops on spider web appearance: a newly named pattern of IgG4 deposition in pemphigus with direct immunofluorescence. Adv Dermatol Allergol 2017; 34: 295-8.

13. Sävervall C, Lćrke Sand F, Francis Thomsen S. Pemphigoid gestationis: current perspectives. Clin Cosmet Investig Dermatol 2017; 10: 441-9.

14. Di Zenzo G, Marazza G, Borradori L. Bullous pemphigoid: physiopathology, clinical features and management. Adv Dermatol 2007; 23: 257-88.

15. Schmidt E, Benoit S, Bröcker EB. Bullous pemphigoid with localized umbilical involvement. Acta Derm Venereol 2009; 89: 419-20.

16. Oliveira JV, Maruta CW, Sousa JX, et al. Clinical and immunological profile of umbilical involvement in pemphigus vulgaris and pemphigus foliaceus. Clin Exp Dermatol 2012; 38: 20-4.

17. Stransky L. Contact pemphigus vulgaris? Contact Dermatitis 1998; 38: 45.

18. Thongprasom K, Suvanpiyasiri C, Adirek S, et al. Nickel-induced oral pemphigus vulgaris-like lesions. Acta Stomatol Croat 2011; 45: 202-8.

19. Peiser M. Role of Th17 Cells in skin inflammation of allergic contact dermatitis. Clin Dev Immunol 2013; 2013: 261037.

20. Nakae S, Komiyama Y, Nambu A, et al. Antigen-specific $T$ cell sensitization is impaired in IL-17-deficient mice, caus- ing sup-pression of allergic cellular and humoral responses. Immunity 2002; 17: 375-87.

21. Timoteo RP, da Silva MV, Miguel CB, et al. Th1/Th17-related cytokines and chemokines and their implications in the pathogenesis of pemphigus vulgaris. Mediators Inflamm 2017; 2017: ID7151285.

22. Dmochowski M, Bowszyc-Dmochowska M. Nadwrażliwość na nikiel a opryszczkowate zapalenie skóry. Dermatol Klin 2007; 9: 275-6.

23. Daneshpazhooh M, Fatehnejad M, Rahbar Z, et al. Traumainduced pemphigus: a case series of 36 patients. J Dtsch Dermatol Ges 2016; 14: 166-71.

24. Vinay K, Kanwar AJ, Saikia UN. Pemphigus occurring at tuberculin injection site: role of cytokines in acantholysis. Indian J Dermatol Venereol Leprol 2013; 79: 539-41.

25. Camisa C, Helm TN, Liu YC, et al. Paraneoplastic pemphigus: a report of three cases including one long-term survivor. J Am Acad Dermatol 1992; 27: 547-53.

26. van Mook WNK, Fickers MM, Theunissen PH, et al. Paraneoplastic pemphigus as the initial presentation of chronic lymphocytic leukemia. Ann Oncol 2001; 12: 115-8.

27. Jimenez A, Hoyer P, Wilkerson M. An atypical presentation of pemphigus vegetans in the umbilicus. SKIN 2020; 4: 452-5.

28. Cetinarslan TS, Türel Ermertcan A, Temiz P, et al. Pemphigus vegetans arising in umbilicus: successful clearance with intralesional steroid. Indian J Dermatol Venereol Leprol 2018; 84: 522.

29. Casuriaga Lamboglia AL, Minut Gubitosi A, Guebenlián Bakerdjian C, et al. Pemphigus vulgaris in pediatrics: a case report. Rev Chil Pediatr 2018; 89: 650-4.

30. Aoki V, Rivitti EA, Diaz LA. Update on fogo selvagem, an endemic form of pemphigus foliaceus. J Dermatol 2015; 42: 18-26.

31. Chams-Davatchi C, Valikhani M, Daneshpazhooh M, et al. Pemphigus: analysis of 1209 cases. Int J Dermatol 2005; 44: 470-6. 